\title{
We Should Search for Extant Life on Mars in this Decade
}

\section{A White Paper Submitted to the 2020 NRC Planetary Science Decadal Survey}

July 14,2020

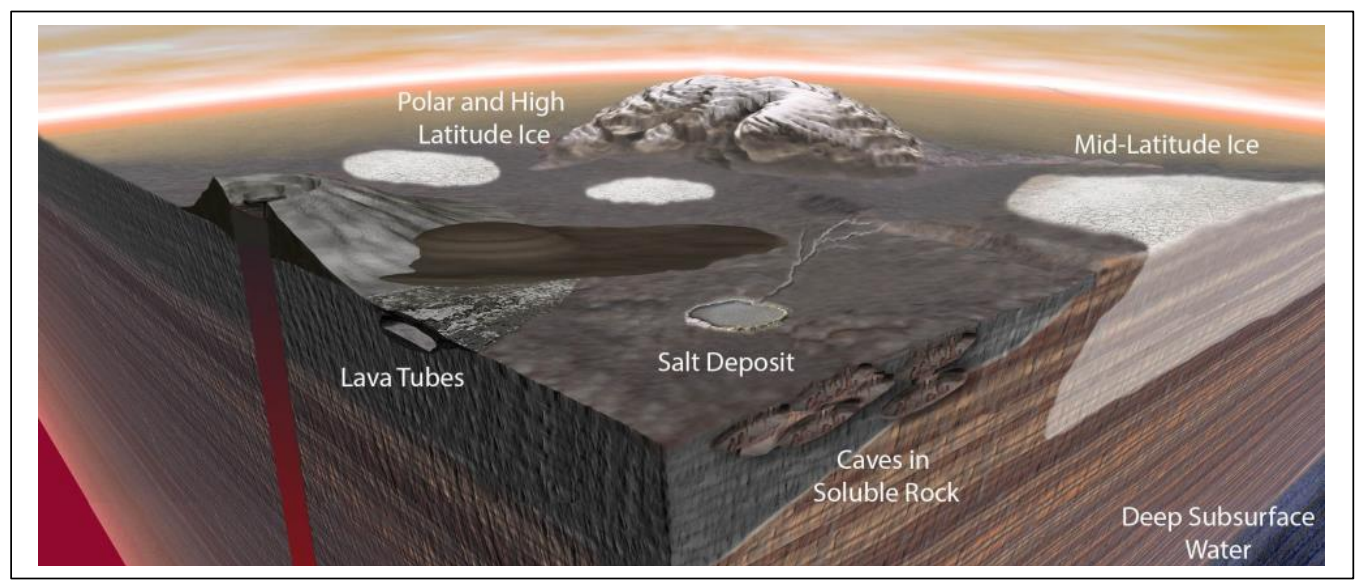

Lead Author: Carol Stoker

NASA Ames Research Center

MS 245-3, Moffett Field, CA 94035

650-604-6490 (Office), 408-666-3044 (Cell)

Email: carol.stoker@,nasa.gov

\section{Coauthors:}

Jennifer G. Blank (Blue Marble Space \& NASA Ames Research Center)

Penelope Boston (NASA Ames Research Center

Luoth Chou (NASA GSFC \& Georgetown University)

Shiladitya DasSarma (University of Maryland)

Jennifer Eigenbrode (NASA Goddard Space Flight Center)

Natalie Grefenstette (Santa Fe Institute)

Diana Northup (University of New Mexico)

Andrew Schuerger (University of Florida)

Dirk Schulze-Makuch (Technical University Berlin)

Vlada Stamenković (Jet Propulsion Laboratory)

Jesse Tarnas (Brown University)

\section{CoSigners:}

65 Cosigners, name list at time of submission is on page 7.

Click here to view cosigner document or paste this link into your browser: https://docs.google.com/spreadsheets/d/11FDuMef3eoXgn_2fFL_c3ka2-

UW'TCQyoAKJ4P4DCpUE/edit?usp=sharing 
Summary: A search for evidence of extant life on Mars should be conducted within the decade. Several potentially habitable environments for life occur on modern Mars. In spite of a vigorous campaign of exploration of the surface over the last 3 decades, no mission has attempted to search for signatures of extant life since the pair of Viking landers touched ground in 1976. In this White Paper, four examples of habitable environments on Mars that merit a search for life are described along with potential approaches for their exploration. These concepts were developed in the recent workshop "Mars Extant Life: What's Next?" (Carrier et al. 2020). We advocate that the search for extant Martian life should be the subject of a focused mission or program in the next decade or be included among New Frontiers candidate missions.

\section{Scientific importance of finding extant life on Mars}

Finding an example of extant life beyond Earth would be one of the greatest scientific discoveries of all time. This is especially important because (once discovered) the biochemistry and metabolism of the life form can be studied. We know, from the noble gas chemistry patterns in meteorites and modeling of impact physics that Earth and Mars exchange materials over geologic time because impacts eject rock and crustal materials into space that are eventually deposited on other planets and moons (Mileikowsky et al. 2000). It seems possible, therefore, that the Earth and Mars could share life with a common origin and similar biochemistry; if this is the case, life on Mars likely experienced billions of years of evolution in isolation from Earth. Alternatively, Mars may host a distinct genesis of life which could be evident from its different biochemistry. Either discovery would rock the world and change, fundamentally, our understanding of life in the solar system and beyond.

Proof of extant life is unlikely to be found from the upcoming Mars sample return mission because the site for sample collection, Jezero Crater, was chosen for its ancient habitability and likelihood to host fossil evidence of life but is deemed not habitable at present. The Mars 2020/Perseverance rover mission to collect samples is just beginning (launch in July 2020), and the mission to return samples will not be completed for at least a decade. Within that period, technology development for human exploration will be underway and it is possible that humans will land on Mars without an updated knowledge of extant life on Mars, which might pose a risk both to those mission crews and to Earth when they return. Thus, it is urgent to perform the search for extant life on Mars prior to human exploration missions.

\section{Viking and the search for life}

The 1976 Viking landers carried the only instruments ever flown that were meant to detect extant life on Mars: three experiments designed to search for metabolic activity in soil samples. Although the soil was very reactive, the measurements were not consistent with known microbial processes on Earth and could be explained by an abiotic oxidized soil. Also, soil microbial life was discounted partly because an organic analyzer, the pyrolysis Gas Chromatograph Mass Spectrometer (GCMS), did not detect organic compounds in the soil even at concentrations that would be expected from meteoritic sources hitting the planet's surface. This finding was puzzling for decades until the 2008 Phoenix Mission discovered that the soil contains up to $2 \%$ perchlorate (Hecht et al. 2008), a highly oxidizing compound. Navarro-Gonzales et al. (2010) proposed that trace levels of chlorinated hydrocarbons detected by the Viking GCMS were due to the reaction of perchlorate and soil organics in the thermal volatilization stage of the analysis. More recently, the Mars Science laboratory mission analyzed Mars samples with GCMS and found indigenous organics at ppm levels in mudstone samples, but results were inconclusive as to whether the soil organics were biogenic or cosmogenic in origin (Eigenbrode et al. 2018). The very low observed concentration of organics, the reactive oxidizing soil hypothesis for the Viking biology experiment results, along with the recognition of surface conditions on Mars being cold and arid by terrestrial standards, led to a scientific consensus that there is no extant life on the Martian surface. However, the abundant morphological evidence that the surface of Mars has been shaped by flowing water influenced post-Viking missions to focus on evidence of past habitable Martian environments and instruments capable of detecting extant life were not included in the vehicle payloads. Even though the current surface conditions on Mars are at the extreme limits of growth of 


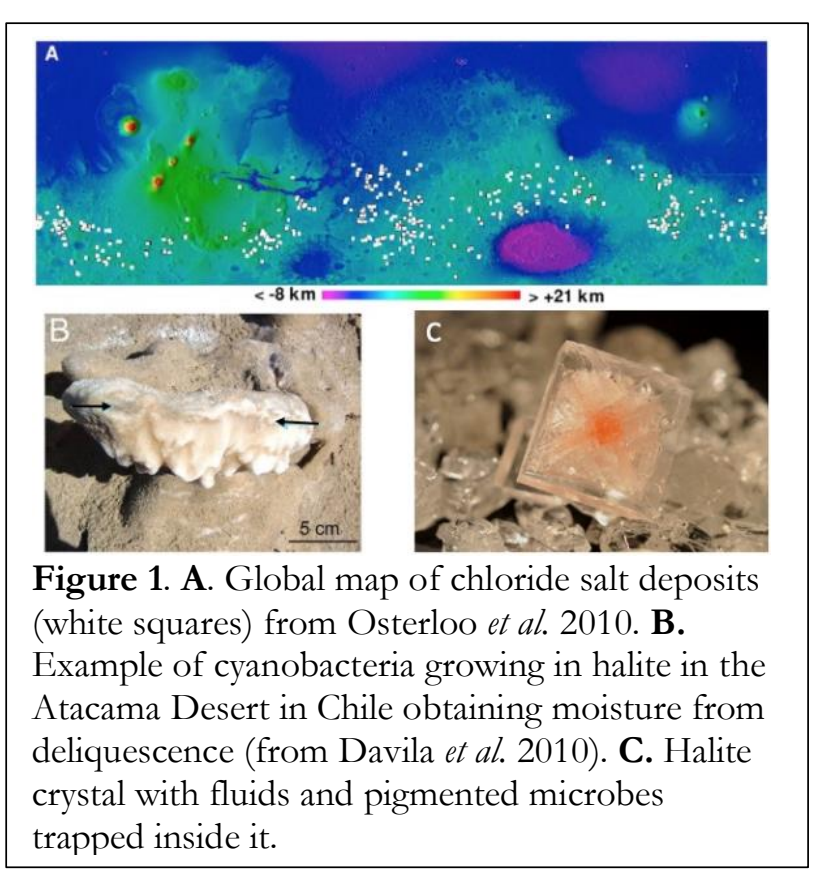

terrestrial life on average, extremophilic Earthlike life may persist on Mars in selected sheltered environments. Examples of potential habitats for life on Mars are described below.

\section{Environments on Mars that may host extant life}

Salts and Evaporites: The properties of evaporates and salts make them highly desirable and easily accessible environments in the search for extant life on Mars (Davila and SchulzeMakuch 2016). On Earth, evaporites and associated brines are inhabited in many places across the globe, supporting a wide diversity of microbial communities including phototrophs, lithotrophs, and heterotrophs (DasSarma and DasSarma 2017). Endolithic phototrophs are found associated with gypsum crusts, and haliteentrapped halophilic archaea and bacteria are commonly observed in enclosed brine fluids, with striking and easily detectable carotenoid pigment biosignatures (DasSarma et al. 2019).

Halite and gypsum minerals offer radiation protection by attenuating ultraviolet light and provide protection from long-term desiccation by deliquescence (Davila et al. 2010). Finally, dissolved salts also extend the temperature range for maintaining liquid water through freezing point depression and by formation of supercooled liquids, expanding the possibility of life processes at subzero temperatures (Toner et al. 2014). Concentrated brines are also common in ice vein networks, due to exclusion of dissolved salts during freezing point depression and ice formation. Furthermore, because many salts are hygroscopic, liquid brines might form near the surface in locations that receive periodic frosts. Such environments can potentially support microbial growth.

Salts and evaporites are common at the surface and near-subsurface of Mars and are readily accessible to mobile platforms. The Thermal Emission Imaging System (THEMIS) has mapped the global distribution of chloride salts on the Martian surface with at least 600 regions identified to date (Osterloo et al. 2010, Figure 1A). Many sites are located in local depressions that may have formed as a result of surface runoff, groundwater upwelling, and/or hydrothermal activity. Examples include: (1) Eastern Margaritifer Terra which exhibits mineral precipitation in upwelling fluids from crater floor fractures (Thomas et al. 2017), and (2) Columbus Crater in Terra Sirenum that possesses groundwaterfed paleolakes and evaporites (Wray et al. 2011).

Sampling evaporite deposits for biosignatures can be done with current shallow drilling technology like that on the Curiosity rover. The Mars 2020 mission will explore deltaic deposits in Jezero crater. If the Perseverance rover encounters and collects evaporite salts that are returned to Earth, then these samples might provide new information on extant life on Mars.

Ice Rich Terrains: These are important locations to search for extant Martian life and are planetary protection "special regions" where higher spacecraft sterilization and cleanliness is required because of their potential habitability (Fairén et al. 2017). Ground ice is accessible and widespread on Mars at latitudes above 35。 N /45。 S (Piqueux et al. 2019, Figure 2A). On Mars, quasiperiodic climate change results from variations in Mars' orbital parameters (e.g. obliquity, eccentricity, and season of perihelion) causing the intensity of incident sunlight at a given latitude to change over time (Laskar et al. 2004, Figure 2B) along with the locations and timing of habitable conditions in the ice.

The subsurface ice at the Phoenix landing site at 68. N may be habitable at high obliquity (Stoker et al. 2010) when the subsurface ice is warm enough (temperatures greater than -20 oC) to support microbial growth down to $\sim 1 \mathrm{~m}$ depth (Zent 2008). Nearly pure ice observed under $\sim 5-10 \mathrm{~cm}$ of dry soil by Phoenix (Figure 2C) may result from freeze-thaw processes that can segregate pure ice from soil (Mellon et al. 2009). While life in the high latitude region sampled by Phoenix should not be 
metabolically active under present climate conditions, its biosignatures could be detected and its recent growth inferred from the young geological age of the ice deposits.

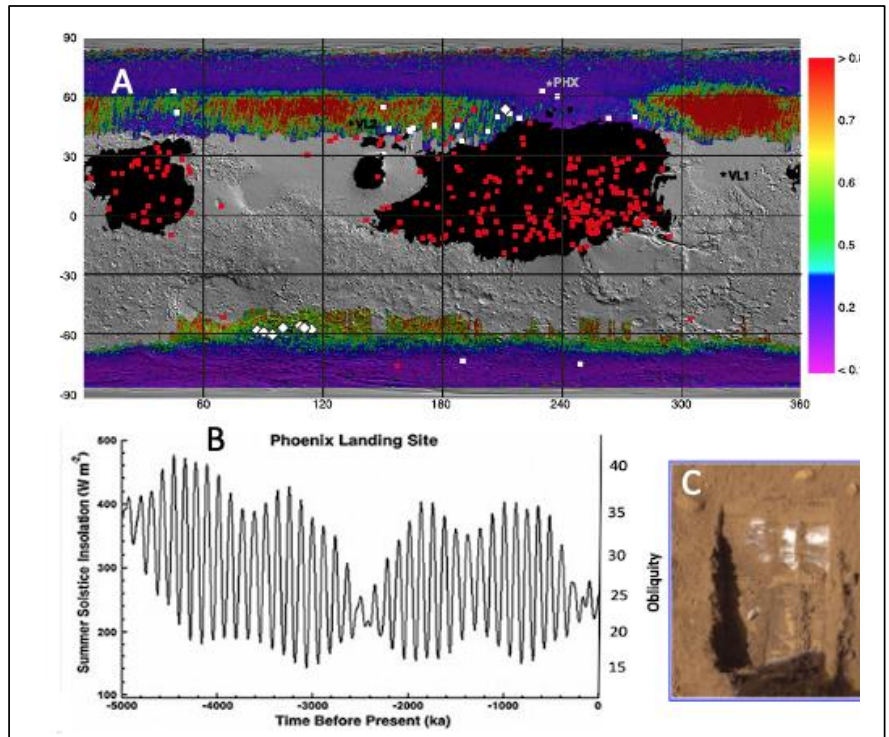

Figure 2. A. Global map of subsurface ice from Piqueux et al. 2019. Colors indicate depth to ice. B. Variation in obliquity and maximum insolation over the last $5 \mathrm{My}$ at the Phoenix landing site. C. Pure ice (white) exposed by digging to $5 \mathrm{~cm}$ depth at Phoenix site.

Transient liquid water can occur on current Mars from the melting of ice (Hecht, 2002); the stability is enhanced when salts are also present forming brines. Current summer temperatures in ice-rich midlatitude regions are sufficient to support life if melting ice or frost provides transient water. Transient liquid water from melted snow supports microbial life residing below the surface of sandstones in Antarctic dry valleys (Sun 2013); analog sites with temperatures comparable to modern midlatitude Mars. It is important to determine if life exists in midlatitude ice prior to human exploration activities that aim to use that resource to prevent both the risk to Earth of inadvertently bringing potentially harmful organisms from Mars, risks to astronaut health, and the complication of recognizing Mars life once terrestrial contamination has occurred.

Sampling ground ice to search for life can be accomplished with landers or rovers carrying 1-2 m drilling systems. McKay et al. (2013) have proposed a Discovery Class mission that would include a stationary lander with a $1 \mathrm{~m}$ drilling system to search for biosignatures of life at the Phoenix Mission landing site. Rover-based drilling would be preferable to search for life in mid-latitudes where the ice depth and heterogeneity below the surface is not well known. Two rover missions with representative drilling capability will be flown in the near future: the lunar Volatiles Investigating Polar Exploration Rover (VIPER) (Colaprete et al. 2019) and the ExoMars rover (Vago et al. 2017). Flight prototype life detection instruments fed with a $1 \mathrm{~m}$ drill similar to that on VIPER have successfully identified biosignatures in Mars analog hyper arid Atacama Desert samples that were collected and analyzed during Mars mission simulations demonstrating that this life detection mission concept can be successful.

Volcanic Caves: Volcanic caves are another high priority environment in the search for extant life on Mars (e.g., Boston et al. 2001; Léveillé \& Datta, 2010), as they protect their interiors from cosmic radiation and energetic solar events, changing surface climatic conditions, and small-scale impact events. Caves can be warmer, wetter, and more protected, therefore more habitable than the surface. More than 1000 candidate caves in volcanic terrain have been identified on Mars from orbital imagery and many occur in the Tharsis volcanic complex area (Figure 3A and B). A cave with natural openings offers direct access to the subsurface, with a relatively stable thermal environment that can persist over geologic time and preserve volatiles (Williams et al. 2010), while voids with no surface openings are detectable via ground penetrating radar and other geophysical methods and provide potential for sealed time capsules with relatively easy drill access. Microbial life in terrestrial volcanic caves that lives on chemical energy derived from limited organic carbon and minerals and is found on and in a wide variety of mineral features, from silica-rich, to carbonate, iron, and other metals which are distinctive from their basaltic host rock (Northup et al. 2011, Fig. 3C and D). Such features preserve microbes extremely well in situ. In some cases, obvious moist biofilms are found in caves but in other cases mineral forms trap and preserve microbial structures that are revealed with microscopy (Boston et al. 2001). A variety of robotic approaches have been proposed to overcome challenges related to 


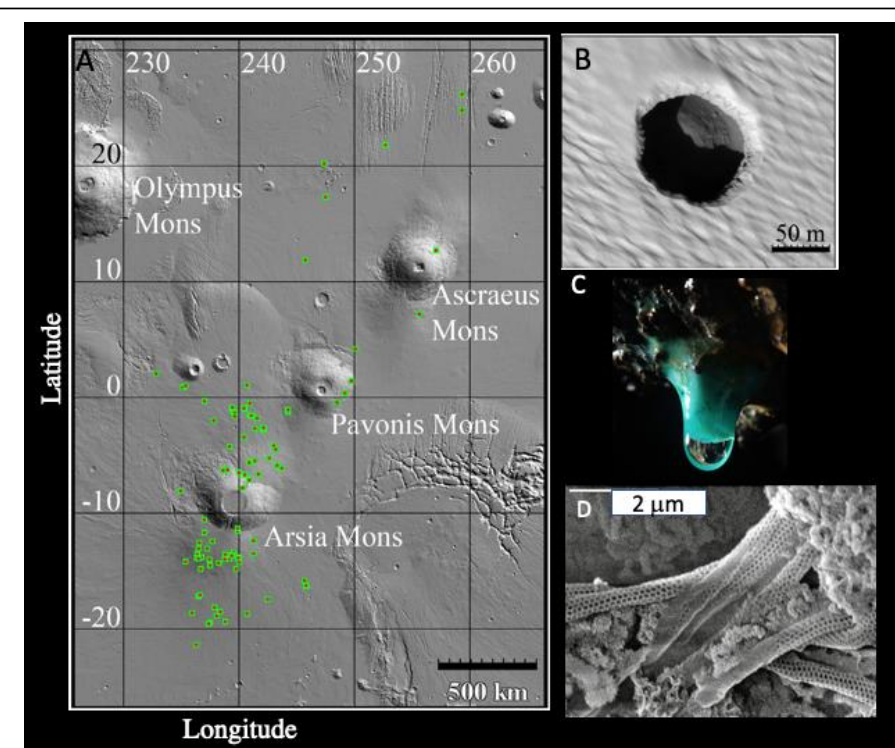

Figure 3. A. Map showing concentration of a portion of more than 1000 candidate cave locations on Mars. B.

Martian pit crater with floor at least $100 \mathrm{~m}$ below the surface. (A, B source: Cushing et al. 2017). C. Turquoise colored mineral deposit in a Hawaiian volcanic cave. D. Scanning Electron Microscope image of microbe structures in the turquoise mineral (C, D source: Northup et al. 2011). autonomous ingress and navigation inside caves including (1) entry from the surface down into the cave system through a skylight, possibly via a large $(>50 \mathrm{~m})$ vertical drop, (2) traversing an irregular floor surface, (3) obstacle avoidance (e.g., collapsed ceiling blocks), (4) operations in darkness, and (5) simultaneous mapping and localization (away from surface communications). Drones can play a role in evaluating habitable conditions in caves and survey for rover access and trafficability. Relevant new technologies, particularly for autonomous navigation and mapping in subsurface environments, are being developed and tested in the current US Department of Defense Advanced Research Projects Agency (DARPA) Subterranean Challenge. These capabilities are directly relevant to the exploration of lunar and Martian caves and pits.

Deep Subsurface: The deep subsurface might be the largest and longest-lived potentially habitable environment on Mars, possibly existing from the Noachian (Michalski et al. 2013; Onstott et al. 2019) until now. This contrasts with environments on the surface and near-subsurface, where consistently habitable conditions declined early in Martian history, as the geologic record shows (e.g. Kite, 2019).

Earth's subsurface is estimated to contain $\sim 1030$ cells, comparable to $\sim 10-60 \%$ of the total biomass of the surface biosphere and likely exceeding the microbial biomass of the surface biosphere (Bar-On et al. 2018; Onstott et al., 2019). Potentially habitable water-bearing deep subsurface environments could host Martian life in chemical and physical conditions similar to those of Earth's deep subsurface (Tarnas et al. 2018). Hypothetical Martian organisms similar to some Earth organisms could survive and thrive in the subsurface of Mars with no significant biological adaptation compared to their earthly counterparts. Biogeochemical processes in the subsurface may support Gibbs free energies conducive to a wide diversity of metabolism types (Figure 4).

Evaluation of deep subsurface habitability could be achieved with surface instruments deployed by small low-cost spacecraft. This includes landed transient electromagnetic sounders under development at NASA JPL to characterize liquid groundwater and/or Tunable Laser Spectrometers to localize released trace gases and determine if they are biosignatures. Physical access to the subsurface would require deep drilling systems. Drills reaching $10 \mathrm{~m}$ have been demonstrated under simulated or Mars analogue conditions and are compatible with New Frontiers class lander missions. Deep $(\sim 100 \mathrm{~m})$ drills are becoming technologically feasible for robotic missions, for example, using a low-mass wireline drill recently demonstrated drilling to $111 \mathrm{~m}$ in Greenland ice (Mellerowicz et al. 2019, Bhartia et al. 2019). Physical access to the subsurface would allow biogeochemical and life detection measurements to be made by a variety of instruments on the surface.

\section{Methods to detect extant life}

Since life on Mars could either stem from a shared ancestry with life on Earth or from a separate genesis, life detection strategies can be either agnostic, meaning they do not depend upon Martian life 


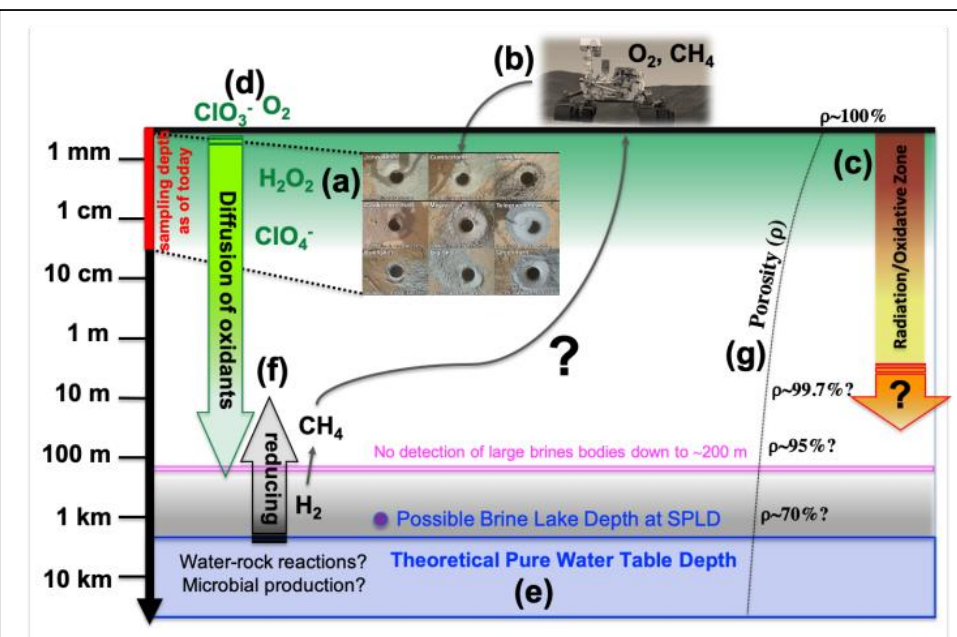

Figure 4. Science with Depth: (a) Variable redox state transition in the upper few $\mathrm{cm}$ as shown by MSL. (b) Atmospheric methane possibly derived from rock-water reactions. (c) Penetration depth of oxidizing and ionizing radiation environment estimates vary from 1-10 m. (d) Oxidants in near-surface environments, possibly linked to $\mathrm{CH}_{4}$ destruction. (e) Depth of "pure" water table is latitude dependent and in the kilometer range. Brines could be locally shallower but have a low water activity close to the surface. (f) Water-rock reactions producing $\mathrm{H}_{2}$ and possibly $\mathrm{CH}_{4}$. (g) Porosity with depth informs the potential for groundwater and subsurface life. Pink denotes the depth of $\sim 200 \mathrm{~m}$ above which large-scale brines seem to be uncommon based on available radar instrument data. A possible brine lake detected using MARSIS instrument radar data (Orosei et al. 2018) is shown at $1.5 \mathrm{~km}$ depth. being similar to terrestrial life, or can be performed under the assumption that life on Mars would be biochemically similar to terrestrial life. A successful search must define features of living systems that should be present in any form of life. One phase of the analyses would be to determine molecular composition, including the complexity, distribution, isotopic composition, enantiomeric and stereoisomeric distribution, and chirality of the compounds present. If looking for familiar life, we can also search for specific compounds such as nucleic acids, fatty acids, amino acids (or more generally: polymers), as well as inorganic signatures such as biominerals. These suites of chemical analyses can potentially be performed using mass spectrometers (MS) (e.g. gas chromatography MS, laser desorption ionization MS and tandem MS) and spectroscopic instruments (e.g. vibrational and fluorescence spectroscopy) that have high heritage in space exploration or have been selected for future missions. Another potential biosignature involves looking for surface complexity, which can be explored through the newly proposed "fingerprinting" method (Johnson et al. 2018) that looks for diversity in oligonucleotide binding sites that could be reflective of a living, informational system.

Another phase of analysis could be focused on the structure and texture of a sample, its morphology, and the potential presence of localized or cellular structures, as well as non-Brownian motility. These studies can be conducted using microscopic techniques (e.g. holographic, atomic force, fluorimetric microscopy, and scanning electron microscopy) and other spectroscopic instruments such as those on board the Mars 2020 rover or other current surface rovers' tools.

Finally, sample analysis could look for the presence of an active metabolism through the consumption or release of compounds (e.g., respirometry, labeled-release experiments), through changes in energy utilization (e.g., micro-calorimetry and electrochemistry), or disequilibrium redox reactions inconsistent with abiotic processes. If looking for familiar life, we can also search for specific trace gases such as methane, $\mathrm{H}_{2}, \mathrm{CO}$, and complex volatile organic compounds (VOCs) at concentrations that are inconsistent with abiotic processes.

The confidence in results indicating life would depend on the ability to distinguish signals from noise and on individual results within the context of the entirety of the results. Future missions would therefore benefit from the development of suites of instruments capable of conclusive in situ detection of extant life. Improvements are needed for sample preparation in order to optimize biosignature detection. Non-invasive methods (e.g. multi-spectral/fluorescence reflectance spectroscopy) can provide potential biological detection evidence without ingestion or preparation. Spacecraft resources 
should support a sufficient number of sample analyses dedicated to replicate measurements, and importantly, positive and negative controls. Contamination control should be coupled with contamination knowledge so that Earth-based material can be eliminated as a possible source of any biological material discovered in Martian samples.

\section{Search for extant life in the coming decade.}

Exploring for life on Mars can be accomplished in the next decade with a dedicated mission or program of missions along the lines of the Mars Scout program. Alternatively, including the search for extant life on Mars as a mission objective in the New Frontiers program would facilitate competition for creative concepts. Finally, there is a pressing need for a focused instrument development program to advance technological approaches needed to search for extant life. A targeted instrument development program with that objective would also benefit the search for life on other solar system objects as well as on Mars.

\section{References}

Bar-On, Y.M. et al. 2020. PNAS 115(25), 6506-6511.

Bhartia, R. et al. 2019. AGU fall meeting Dec. 2019, San Francisco, CA.

Boston, P.J. et al. 2001. Astrobiology 1(1), 25-55.

Carrier, B.L. et al. 2020. Astrobiology 20(6).

Colaprete, A., et al. 2019. AGU fall meeting Dec. 2019, San Francisco, CA.

Cushing, G.E. et al., 2015. J. Geophys. Res. Planets 120, 1023-1043.

DasSarma, S. et al. 2017. Encyclopedia of Life Science, Wiley.

DasSarma, S. et al. 2020. Extremophiles 24, 31-41.

Davila, A.F. et al. 2010. Astrobiology 10, 617-628.

Davila, A.F. and Schulze-Makuch, D. 2016. Astrobiology 16(2), 159-168.

Fairén, A.G. et al. 2017. Astrobiology 17(10), 962-970.

Hecht, M.H. 2002. Icarus 156, 373-386.

Hecht, M.H. et al. 2009. Science 325, 64-67.

Johnson, S.S. et al. 2018. Astrobiology 18(7), 915-922.

Kite, E.S. 2019. Space Science Reviens 215(1).

Laskar, J. et al. 2004. Icarus 170, 343-364.

Léveillé, R.J. et al. 2010. Planet Space Sci. 58(4), 592-598.

Magnabosco, C. et al. 2018. Nature Geoscience 11(10), 707-717.

McKay, C.P. et al. 2013. Astrobiology 13(4), 334-353.

Michalski, J.R. et al. 2013. Nature Geoscience 6(2), 133-138.

Mellerowicz, B. et al. 2019. AGU fall meeting Dec. 2019, San Francisco, CA.

Mileikowsky, C. et al. 2000. Icarus 145, 3910427.

Mellon, M. et al. 2009. J. Geophys. Res. 114, E00E07.

Navarro-Gonzalez, R. et al. 2010. J. Geophys. Res. 115 (E12): E12010.

Northup, D.E. et al. 2011. Astrobiology 11(7).

Onstott, T.C. et al. 2019. Astrobiology 19(10), 1230-1262.

Orosei, R. et al. 2018. Sci. 361, 190-193.

Osterloo, M.M. et al. 2010. J. Geophys Res. 115:E10012.

Piqueux, S. et al. 2019. Geophys. Res. Lett. 46, 14290-14298.

Sun, H.J. 2013. Biology 2, 693-701.

Stoker, C. R. et al. 2010. J. Geophys Res. 115, E00E20.

Tarnas, J. D. et al. 2018. Earth and Planetary Science Letters 502, 133145.

Thomas, R. J. et al. 2017. Geophys. Res. Lett. 44, 6579-6588.

Toner, J.D. et al. 2014. Icarus 233, 36-47.

Vago, J.L. et al. 2017. Astrobiology 17(6-7), 471-510.

Williams, K.E. et al. 2010. Icarus 209: 358-368.

Wray, J. et al. 2011. J. Geophys Res 116 E01001.

Zent, A. 2008. Icarus, 196(2), 385-408. 
Last Name, First Name, Affiliation

Alkemade, Sarah, Monash University, Australia

Andersen, Dale T., SETI Institute

Azua-Bustos, Armando, Centro de Astrobiologia (CSIC-INTA), Madrid

Bakermans, Corien, Pennsylvania State University

Baxter, Bonnie K., Westminster College, Utah

Bell, Mary Sue, Jacobs@NASA/Johnson Space Center

Bolivar-Torres, Hermes H., Universidad Nacional Autonoma de Mexico

Bruner, Bob, Denver Museum of Nature and Science

Bywaters, Kathryn, SETI Institute

Cabrol, Nathalie A., SETI Institute

Carrier, Brandi, Jet Propulsion Laboratory

Clark, Benton C., Space Science Institute

Cockell, Charles, University of Edinburgh

DasSarma, Priya, University of Maryland,

Baltimore

Engelhart, Aaron, University of Minnesota

Ertem, Gözen, SETI Institute

Fairén, Alberto G., Centro de Astrobiologia (CSIC-INTA), Madrid

Ferl, Rob, University of Florida, Gainesville FL

Foster, Jamie S., University of Florida

Gasda, Patrick J., Los Alamos National

Laboratory

Green, James H., Texas Tech University

Harris, Rachel, Harvard University

Headrick, Erika, University of Florida, Merritt Island, FL

Huang, Xinchuan, SETI Institute

Jha, Devanshu, MVJ College of Engineering, India

Jones, Daniel S., New Mexico Tech.

Khuller, Aditya, Arizona State University

Kieft, Thomas L., New Mexico Institute of

Mining and Technology

Koeppel, Ari, Northern Arizona University

Lanza, Nina, Los Alamos National Laboratories

Lau, Graham, Blue Marble Space Institute of Science

Laye, Victoria, University of Maryland, Baltimore
Lee, Peter, Hollings Marine Lab, College of Charleston

Li, Xiang, NASA-GSFC \& University of Maryland

Lynch, Kennda, Lunar and Planetary Institute

Mancinelli, Rocco, NASA Ames Research Center

Mckay, Christopher P., NASA Ames Research Center

Millan, Maëva, NASA-GSFC \& Georgetown University

Nicholson, Wayne, U. Florida

Onstott, T.C., Princeton University

Parro, Victor, Centro de Astrobiologia (CSICINTA), Madrid

Paul, Anna-Lisa, University of Florida, Gainesville

Pozarycki, Chad, NASA-GSFC

Ricca, Alessandra, SETI Institute

Rivera-Valentín, Edgard G., Lunar and

Planetary Institute

Roussel, Anais, Georgetown University

Schneegurt, Mark, Wichita State University

Schwartz, James S.J., Wichita State University

Schwender, Petra, University of Florida

Schwieterman, Edward, University of

California, Riverside

Scuderi, Louis , University of New Mexico

Shkolyar, Sventlana, NASA Goddard Space

Flight Center

Skok, J.R., SETI Institute

Smith, Heather, NASA Ames Research Center

Smith, Peter, University of Arizona

Sneed, Evan L., Penn State University

Spry, J. Andy, SETI Institute

ten Kate, Inge Loes, Utrecht University, the

Netherlands

Wade, Brian D., Michigan State University

Warren-Rhodes, Kimberley, SETI Institute

Whyte, Lyle, McGill University, Canada

Wiens, Roger, Los Alamos National Laboratory

Williams, Amy, University of Florida

Wynne, J. Judson, Northern Arizona University 